\title{
Challenges Faced by the Intensive Care Unit during a Terrorist Attack: The Riyadh Experience
}

\author{
Abdullah Al-Shimemeri \\ College of Medicine, King Saud bin Abdulaziz University for Health Sciences, P.O. Box 22490, Riyadh 11426, Saudi Arabia \\ Correspondence should be addressed to Abdullah Al-Shimemeri, aftercom@yahoo.com
}

Received 24 June 2012; Accepted 1 August 2012

Academic Editors: A. Pazin-Filho and C. Roberto

Copyright ( $) 2012$ Abdullah Al-Shimemeri. This is an open access article distributed under the Creative Commons Attribution License, which permits unrestricted use, distribution, and reproduction in any medium, provided the original work is properly cited.

Background. Globally, we are facing a "terror epidemic" with incidences of terrorist attacks on innocent civilians increasing worldwide. The volume and nature of injuries resulting from such attacks challenge even the most sophisticated and well-prepared trauma systems and health institutions. Over seventy percent of terror attacks involve bomb explosions with improvised explosive devices (IEDs) resulting in a unique pattern of injuries to the victims. Here, we present data on the terrorist attacks on the city of Riyadh, Saudi Arabia, between March 1991 to the present and discuss in retrospect the event outcomes and features which may be useful for the preparedness of the intensive care unit (ICU) in the wake of future attacks. Methods. Data from nine hospitals in Riyadh that catered to patients during terrorist strikes were collected retrospectively. Details of the incidents were collected from news media archives. Results. The majority of terror strikes $(\sim 80 \%)$ involved a bomb blast with the use of explosives. The victims included 148 casualties, 45 hospitalizations, 103 discharges after first aid, and 3 incidences of admission to the critical care unit. Conclusion. Using the Riyadh experience, we analyze data and the series of events in the ICU following terrorist explosions. The analysis provides important insights for the trauma unit so that it is better prepared and organized for such incidents in the future.

\section{Introduction}

The majority of the terror incidents around the world involve the use of explosives in carrying out a blast. Public locations with high populations are targeted for carrying out terror bombings. Terrorism attacks in the civilian population are associated with high casualty rates because protections such as body armor employed in combat situations are absent. Terror bombings are characterized by a sudden upsurge in demand for medical care leading to triage complication. Most surgeons and caregivers operate in a nonmilitary environment where a medical response to bomb explosions is often beset with a lack of surgical personnel with adequate exposure to triage in multiple casualty incidents (MCIs). The current wave of terrorism attacks has made it imperative that the trauma response team is adequately prepared. In this work, various aspects of the response of the clinical community to terror bombings are examined with a view to identifying issues that impact the effective management of terror attack victims.
Blast injuries are classified as primary, secondary, tertiary, and quaternary. The most common cause of death is secondary blast injuries, inflicted by flying debris generated by the explosion.

The effects of bomb explosions are generally correlated with the type of blast injuries resulting therefrom. These effects, as described by Bhatoe in 2008, can be broadly classified as blast wind and secondary blast overpressure effects, fragmentation effects, and incendiary thermal effects [1]. Explosives containing trinitrotoluene, nitroglycerin, and other highly explosive substances are associated with very high overpressure (shock wave) generation, according to Bhatoe [2], on a millisecond timescale. The resulting rapid expansion following an improvised explosive device (IED) explosion generates a differential pressure that compresses the surrounding air into a high-energy (HE) shock wave front, quickly followed by regions of subatmospheric pressure levels [3]. The propagation of the emerging blast wave involves spalling implosion and rapid acceleration processes transmitted in the surrounding air [1]. Blast waves are strongly 
amplified in closed spaces and in dense media like water. Airfilled organs, especially the pulmonary, gastrointestinal, and auditory systems, are the most affected by the generated blast winds [2]. This category of injuries is referred to as primary injuries. Also known as barotrauma, they result from cycles of contraction-expansion beyond the affected tissues' natural limit [4]. The severity of primary blast injuries depends on factors such as the proximity of the victim to the detonated IED, the degree of confinement (open versus close space), and the density of the medium (air versus water) [5]. The closer a victim is to the epicentre of blast overpressure, the greater the severity of the blast injuries sustained [6]. Ultraconfined spaces (e.g., buses) are associated with low survivability and high fatality rates as demonstrated in a forensic analysis of the UK terror bombing experience $[3,7]$. Reflection of the short-lived incident shock waves in confined spaces results in a complex blast wave pattern amplifying both the blast energy and lifetime [3, 8]. Amplifications up to 8-9 times are possible in reflected blast wind usually with more devastating consequences resulting both from amplification effect and longer exposure to the generated complex HE blast waves [9-11]. The London trains and bus bombings of 2005, with 50 casualties and 700 injuries, are an example of how confinement significantly enhances the blast wind [6].

Secondary blast injuries result from the fragmentation effect of IEDs. The rapidly expanding shock wave propels fragments from bomb casing, nails, bolts, and metal pellets intentionally included to kill as many people as possible and to increase the injury rate by primary fragmentation [12]. Substances picked up at the site of explosion (secondary fragmentation) such as broken glasses, pieces of fabric, or even human tissue (e.g., from suicide bombers' bodies) are also part of the penetrants inflicting secondary blast injuries. The energised projectiles have been reported to travel at speeds as high as $820 \mathrm{~m} / \mathrm{s}$ inflicting blunt and penetrating trauma type injuries in people within the range of the blast [6]. Life-threatening haemorrhage, gross bodily mutilation, and serious contaminations are common features of this category of blast injuries [13] arising from tissue penetration of indiscriminately flung debris, traditionally called shrapnel after its inventor Henry Shrapnel $[2,4]$. The other aspect of traumatic amputation involves tertiary blast injuries caused by the blast wave propelling the victims against structures, such as walls, floor, and vehicle parts, occurring at the site of explosion [3]. Fractures, head injuries, severe body disruption, and traumatic amputation are common in this category of blast injuries [3,12]. Spinal cord damage is likely when detonation occurs inside a bus, where victims are propelled upward against the vehicle roof, axially compressing the vertebral column. Tertiary blast injuries also include structural collapse traumas usually associated with high mortality rates. According to Arnold et al., mortality rates in terror bombings involving structural collapse (25\%) are significantly higher than mortalities recorded in indoor explosions without structural collapse (8\%) and outdoor explosions (4\%) [14]. This may be related to the difficulty and delay associated with evacuating victims in structural collapse [3].
The thermal incendiary effect and the emission of toxic gases in terror bombings inflict a fourth category of injuries called quaternary blast injuries. Examples are burns resulting both from primary thermal effect of IED detonation and from secondary fires [12]. Aggravation of existing medical conditions, exposure to biological pathogens, toxic gases, and radiation also come under this category of blast injury. Some authors have also included injuries from structural collapse in this group [12].

Incidents of terror bombings in Saudi Arabia began 13 years ago in March 1991, in the aftermath of the second Gulf War and the Iraqi occupation of Kuwait, as a protest against the intervention and deployment of US troops on Saudi streets.

\section{Material and Methods}

This is a retrospective cohort study of all people injured through terrorist acts in the terror bombing incidents in Riyadh, Saudi Arabia. The study was undertaken with the aim of forming preparedness guidelines for the Saudi medical fraternity in the wake of such disasters. Included in this study are all casualty admissions to hospitals, in-hospital deaths, and transfers to critical care units following the terror strikes in Riyadh from 1991 to date. The list of hospitals in Riyadh, Saudi Arabia where patients were admitted following a terror strike, include King Fahad National Guard Hospital (121 cases); Kingdom Hospital (18 cases), King Khalid University Hospital (12 cases), Dallah Hospital (11 cases), Al Hammadi Hospital (8 cases), Mishari Hospital (4 cases), Insurance Hospital (2 cases), Saudi German Hospital (8 cases), and Riyadh Medical Complex (1 case). The data provided here is taken from the intensive care unit of King Fahad National Guard Hospital (KFNGH), which received most of the cases. The intensive care unit at KFNGH is the only Level I trauma center in Saudi Arabia.

Data Collection. Data on patients were obtained from a retrospective review of medical records. Demographic information, injuries sustained, complications, and event outcome data were obtained. Information regarding the events surrounding the terrorist attacks was obtained from news media archives.

\section{Results}

3.1. Incidence. Table 1 lists the major terrorist attacks that occurred in Saudi Arabia from March 1991 to the present. Figure 1 compares the percentage of attacks that involved explosives to those that involved gunshots. Out of 19 incidents of documented terror strikes in Saudi Arabia that were utilized for this study, $15(\sim 80 \%)$ involved the use of explosives, with resultant blast (Figure 1).

3.2. Population. Terrorist acts were aimed mostly at the civilian population and took place in the form of explosions where people congregate in cars. Blasts in residential complexes took heavy tolls on life, including the lives of children. 
TABLE 1: Incidents of terrorist-related violence and bombings in Saudi Arabia in 13 years.

\begin{tabular}{|c|c|c|c|c|}
\hline Year & No. of causalities & No. of persons hospitalized & Place & Mode of attack \\
\hline March 1991 & - & 3 & Jubail & Gunshots \\
\hline November 1995 & 5 & 60 & Riyadh & Car bomb \\
\hline June 1996 & 19 & 386 & Khobar & Explosives in a bus \\
\hline November 2000 & 1 & 1 & Riyadh & Car bomb \\
\hline November 2000 & - & 3 & Riyadh & Car bomb \\
\hline December 2000 & - & 1 & Riyadh & Car bomb \\
\hline March 2001 & - & 2 & Riyadh & Explosion \\
\hline May 2001 & - & 1 & Riyadh & Parcel bomb \\
\hline October 2001 & 2 & 6 & Khobar & Car bomb \\
\hline June 2002 & 1 & - & Riyadh & Car bomb \\
\hline September 2003 & 1 & - & Riyadh & Car bomb \\
\hline January 2003 & 1 & 3 & Riyadh & Gunshots \\
\hline February 2003 & 1 & - & Riyadh & Gunshot \\
\hline May 2003 & - & 1 & Jubail & - \\
\hline May 2003 & 35 & & Riyadh & Bomb explosion \\
\hline May 2003 & 29 & 194 & Riyadh & Bomb explosion \\
\hline November 2003 & 17 & 122 & Riyadh & Bomb explosion \\
\hline April 2004 & 4 & 148 & Riyadh & Bomb explosion \\
\hline May 2004 & 20 & 194 & Riyadh & Car bombs \\
\hline
\end{tabular}

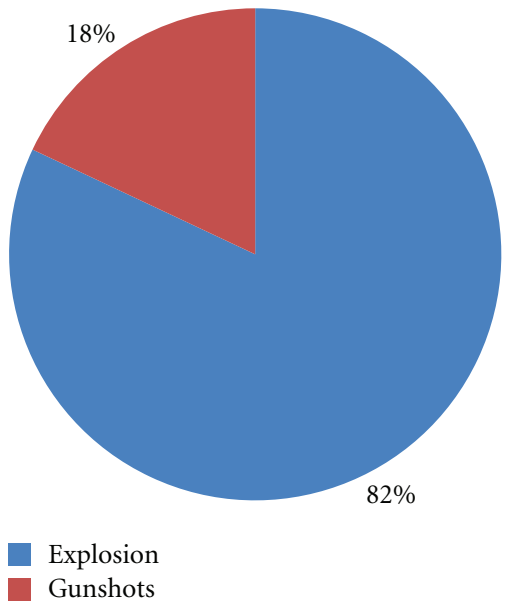

Figure 1: A pie diagram showing the share of blast incidents to total incidents of terrorism. Figures are in percentages.

3.3. Event Outcomes. According to records taken from King Fahad National Guard Hospital intensive care unit, a total of 148 casualties resulted from terror incidents. Forty-five people brought in after the incident required hospitalization. 103 were discharged after first aid, and 3 patients required admission to the critical care unit (Figure 2).

3.4. Arrival to the Hospital. Apart from the usual flow of patients, following a blast in a civilian area, the intensive care unit (ICU) can expect approximately four patients per event for admission. Overall, patients injured in terrorist attacks were more likely to arrive by ambulance. The arrival

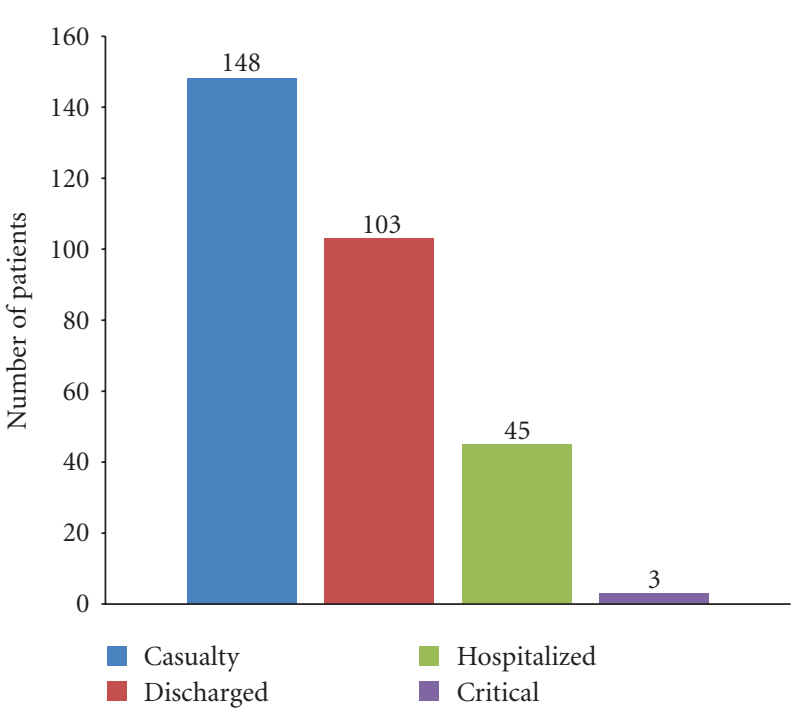

FIGURE 2: Event outcomes for patients brought into the various hospitals in Riyadh after terrorist blasts.

of the first ambulance to the hospital occurs typically about 20 minutes after the attack. There is a continual flow of ambulances from the scene to the hospitals for about 30 minutes after the attack is reported. Thus, the one hour following the blast is crucial for the organization of hospital resources and deployment of medical personnel. Moreover, it was found that patients arrived via various modes of transport. Most of the patients arrived at the hospital in ambulances from the emergency medical service. Some patients arrived in the ICU soon after the event because they needed extensive stabilization prior to surgery. The 
main problems faced were those of manpower, lack of bed space, shortage of blood products, and personnel involved in decision making.

\section{Discussion}

The specific nature and volume of injuries due to terror attacks mandates major disaster preparedness. Since the Kingdom of Saudi Arabia annually hosts mass congregations such as the holy pilgrimage of Haj, it is imperative that the medical fraternity remains ready to face large-scale emergency situations. In this study, we found that the first hour following a blast is crucial for optimum management and allocation of resources so that the number of casualties can be brought down to minimum. (Just a note: did you find this out in this study, or do you know this as it is the "golden hour". I do not see the data in this study that compares outcomes for patients treated within an hour to those treated later.) Terrorist blasts are associated with a high mortality rate due to the serious nature of injuries incurred.

The occurrence of multiple blast injuries (MBIs) with severe injuries involving multiple organs is an important aspect of terror bombing attacks. It represents a combination of different blast injuries, the severity of which depends on factors such as proximity to the detonation site, extent of confinement, and the involvement of structural collapse [15]. Clinical intervention for MBIs proceeds by established guidelines for specific injuries. MBIs involving only one or two victims may not be too problematic to manage but may become highly challenging as the number of casualties increases. Explosions in highly populated locations usually result in MCIs which quickly stretch trauma response beyond its limits of adequacy [16]. A crucial problem resulting directly from casualty volume in MCI is that of triage. The urban setting characterizing terror bombings and the associated injury presentation pattern differentiates MCI traumas from conventional traumas. The extreme severity of MCIs demands that a battlefield attitude to be assumed by trauma response units in triaging efforts [1]. Triage decisions are strongly affected by the supply of resources, especially medical personnel with adequate training and experience with MCI trauma management and medical resources. Compared with a management principle that focuses resources on individual patients in conventional trauma, triage in MCI is geared towards stabilizing as many patients as possible and requires efficient and timely resource allocation. Triage in MCI is characterized by a period of acute resource shortage, when both personnel and medical supplies are momentarily overwhelmed by the volume of casualties. Either overtriage (exhaustion of resources on less significant injuries, or on few patients while neglecting others) or undertriage (withholding critical treatment because of the volume of MCI) then becomes highly probable. It is imperative that a senior surgical officer, with experience in MCI triaging, takes charge of triage decisions. Clinical decision making involving patient evaluation, and interventional measures must be conducted rapidly. Surgeons experienced in matching injuries with cause and required treatment options make them well suited for this role [1].
For efficient triage, the trauma response team must maintain effective communication with nearby hospitals to facilitate and expedite evacuation to their emergency units. It is important that established triage guidelines are followed to ensure efficient distribution to surrounding medical centers and avoid over/undertriage associated with saturating few centers, likely to be proximal to the bombings. Some lifethreatening injuries will usually require immediate intervention and the trauma response units must be ready to provide resuscitative care both at the site of explosion and during evacuation. Kashuk et al. suggested a phased evaluation approach in which resource utilization, evacuation procedures, and triage are guided by a high level of order [1]. At the emergency units of receiving hospitals, evaluation of injuries should be accorded the same degree of urgency. Once patients' injuries are stabilized, they should be transferred to other units to free space for newly arriving casualties. The observed pattern of death in MCIs may also inform how triage is conducted, starting with the initial response, where reduction in number of deaths is the main aim to admission at the ICU where deaths may still result from multiple organ failure [17].

Another long-term problem faced after terror attacks is that the survivors, family members of victims, and witnesses to the scenes of carnage may carry a heavy psychiatric burden in the form of posttraumatic stress disorder (PTSD) [18].

\section{Conclusion}

Victims of civilian bombing attacks are more severely injured compared with other trauma victims and have a pattern of injury that is unique to blast victims. Moreover, a large number of them require critical care; therefore, it is necessary that hospitals follow a formal protocol in dealing with incidents involving mass causalities. Dealing with such incidents not only requires specific medical knowledge but also administrative preparedness to ensure that the limited resources of the ICUs are optimally utilized and that patients are provided with the best possible treatment.

With the incidence of terror bombings becoming an ugly reality that most of the population worldwide has learnt to live with, it is imperative that all medical personnel and ICUs are aware of and prepared for the challenges of facing such a situation. It is the author's sincere hope that this information will not be needed in any other part of the world.

\section{Abbreviations}

ICU: Intensive care unit

MCIs: Multiple casualty incidents

MBIs: Multiple blast injuries

IED: Improvised explosive device

HE: High-energy.

\section{References}

[1] J. L. Kashuk, P. Halperin, G. Caspi, C. Colwell, and E. E. Moore, "Bomb explosions in acts of terrorism: evil creativity challenges our trauma systems," Journal of the American College of Surgeons, vol. 209, no. 1, pp. 134-140, 2009. 
[2] H. S. Bhatoe, "Blast injury and the neurosurgeon," The Indian Journal of Neurotrauma, vol. 5, no. 1, pp. 3-6, 2008.

[3] S. E. Harrisson, E. Kirkman, and P. Mahoney, "Lessons learnt from explosive attacks," Journal of the Royal Army Medical Corps, vol. 153, no. 4, pp. 278-282, 2007.

[4] C. Stevenson, "Evolving mechanisms and patterns of blast injury and the challenges for military responders," Journal of Military and Veteran's Health, vol. 17, no. 4, pp. 17-21, 2009.

[5] A. Mayo and Y. Kluger, "Blast-induced injury of air-containing organs," ADF Health, vol. 7, pp. 40-44, 2006.

[6] A. Gean, "Battlefield brain injury: the lessons from Iraq," 2007, http://www.medicexchange.com/.

[7] G. J. Cooper, R. L. Maynard, N. L. Cross, and J. F. Hill, "Casualties from terrorist bombings," Journal of Trauma, vol. 23, no. 11 , pp. 955-967, 1983.

[8] I. G. Cullis, "Blast waves and how they interact with structures," Journal of the Royal Army Medical Corps, vol. 147, no. 1, pp. 16-26, 2001.

[9] M. A. Mayorga, "The pathology of primary blast overpressure injury," Toxicology, vol. 121, no. 1, pp. 17-28, 1997.

[10] D. Leibovici, O. N. Gofrit, M. Stein et al., "Blast injuries: bus versus open-air bombings-a comparative study of injuries in survivors of open-air versus confined-space explosions," Journal of Trauma, vol. 41, no. 6, pp. 1030-1035, 1996.

[11] E. Katz, B. Ofek, J. Adler, H. B. Abramowitz, and M. M. Krausz, "Primary blast injury after a bomb explosion in a civilian bus," Annals of Surgery, vol. 209, no. 4, pp. 484-488, 1989.

[12] C. T. Born, "Blast trauma: the 4 th weapon of mass destruction," Scandinavian Journal of Surgery, vol. 94, no. 4, pp. 279 $285,2005$.

[13] D. Chandler, "Blast-related ear injury in current U.S. military operations: role of audiology on the interdisciplinary team," ASHA Leader, vol. 11, no. 9, pp. 8-29, 2006.

[14] J. L. Arnold, P. Halpern, M. C. Tsai, and H. Smithline, "Mass casualty terrorist bombings: a comparison of outcomes by bombing type," Annals of Emergency Medicine, vol. 43, no. 2, pp. 263-273, 2004.

[15] A. Gawande, "Casualties of war-military care for the wounded from Iraq and Afghanistan," New England Journal of Medicine, vol. 351, no. 24, pp. 2471-2475, 2004.

[16] D. Leibovici, O. N. Gofrit, and S. C. Shapira, "Eardrum perforation in explosion survivors: is it a marker of pulmonary blast injury?" Annals of Emergency Medicine, vol. 34, no. 2, pp. 168-172, 1999.

[17] J. Tintinalli, J. Stapczynski, O. J. Ma, D. Cline, R. Cydulka, and G. Meckler, Tintinalli's Emergency Medicine: A Comprehensive Study Guide, 7th edition, 2011.

[18] S. H. Goh, "Bomb blast mass casualty incidents: initial triage and management of injuries," Singapore Medical Journal, vol. 50, no. 1, pp. 101-106, 2009. 


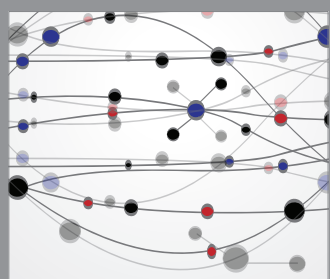

The Scientific World Journal
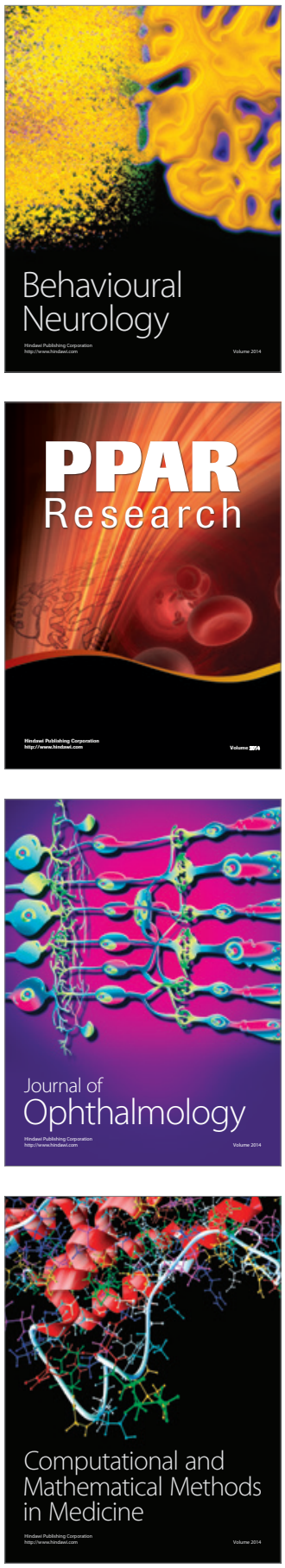

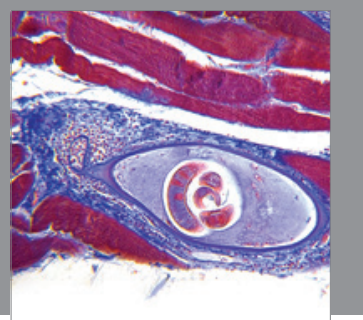

Gastroenterology

Research and Practice
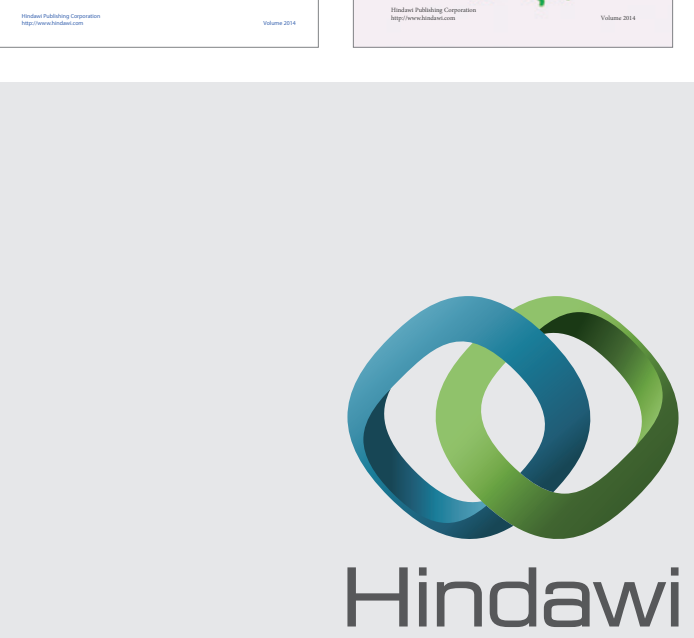

Submit your manuscripts at

http://www.hindawi.com
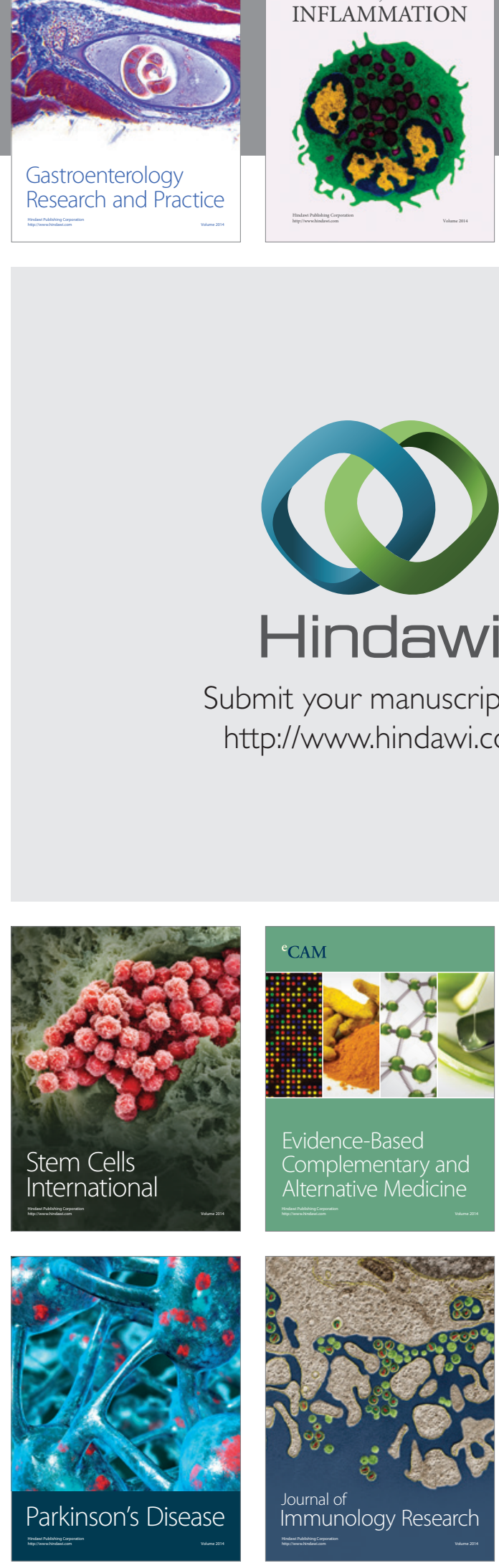

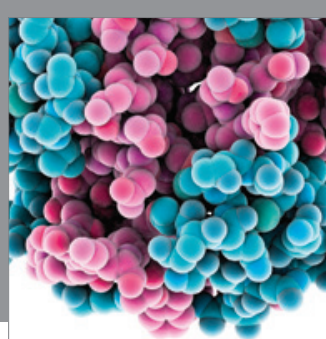

Diabetes Research
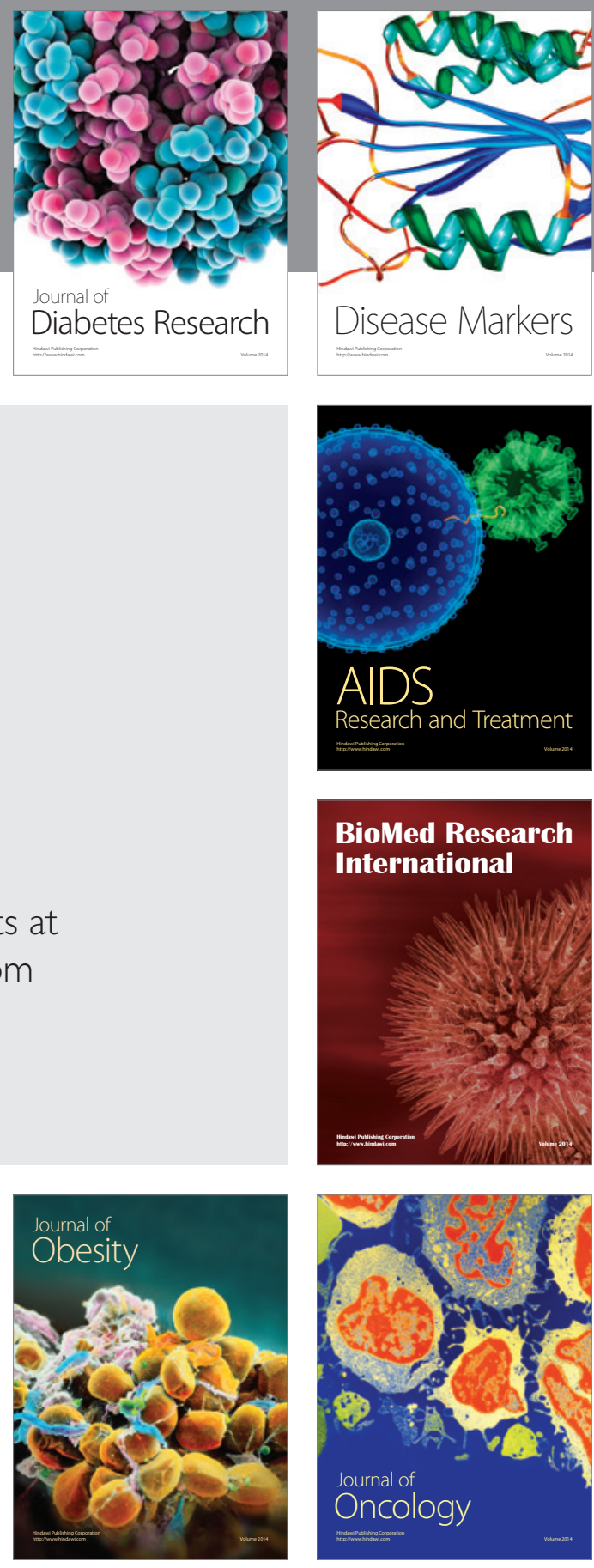

Disease Markers

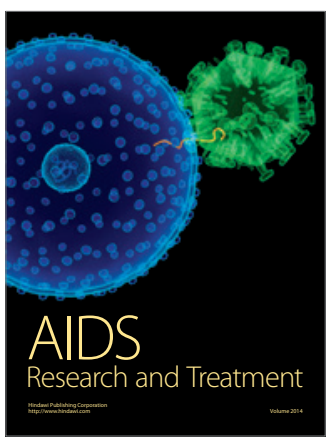

BioMed Research

International
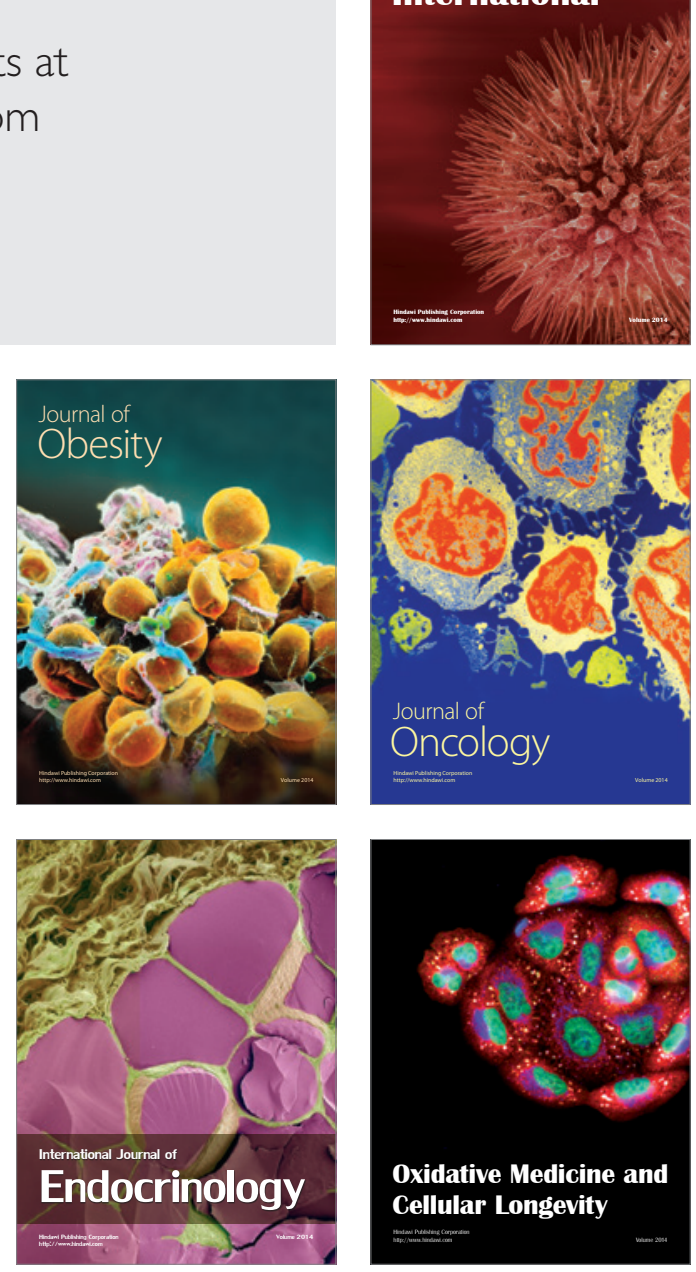